\title{
PeRfil de saúde dos trabalhadores de UM Centro de saúde-escola ${ }^{1}$
}

Lucila Castanheira Nascimento ${ }^{2}$ Iranilde José Messias Mendes ${ }^{3}$

Nascimento LC, Mendes IJM. Perfil de saúde dos trabalhadores de um Centro de Saúde-Escola. Rev Latino-am Enfermagem 2002 julho-agosto; 10(4):502-8.

O estudo buscou descrever o perfil de saúde dos trabalhadores (184) de um Centro de Saúde-Escola de Ribeirão Preto, sob a perspectiva da Promoção de Saúde, modelo epidemiológico de Blum e fatores de risco para doenças crônico-degenerativas. Foi realizado estudo descritivo utilizando-se entrevista estruturada. Concluiu-se que os trabalhadores eram predominantemente do sexo feminino, faixa etária de 35 a 39 anos e escolaridade "superior completo". Dentre os achados, constatou-se que 61,9\% tinham sobrepeso e obesidade; a hipertensão foi detectada em 33,1\%; 41 (22,3\%) apresentaram pressão arterial acima do nível da normalidade e a obesidade mostrou-se presente em mais da metade dos indivíduos com pressão arterial alterada. Os dados possibilitaram explorar riscos autocriados para as doenças crônico-degenerativas; identificar fatores relacionados à promoção de saúde e prevenção de doenças e refletir sobre o autocuidado e auto-responsabilidade, visando à qualidade de vida que desfrutarão, em decorrência da transição demográfica e epidemiológica que vivenciamos.

DESCRITORES: enfermagem em saúde pública, promoção da saúde, trabalhador de postos de saúde

\section{heAlth PROfile Of WORKers in A teAching heALth CENTER}

The purpose of this study was to describe the health profile of the 184 workers employed in a Teaching Health Center in the city of Ribeirão Preto from the viewpoint of Health Promotion, using Blum's Epidemiological Model and the risk factors related to chronic-degenerative diseases. A descriptive study was carried out using a structured interview. The authors of the study concluded that the workers were mainly females at the age range of 35 to 39 years with a college degree. The results showed that $61.9 \%$ were overweight or obese; hypertension was detected in $33.1 \%$; 41 (22.3\%) had blood pressure above normal levels and obesity was present in more than half of the individuals with altered blood pressure. The data enabled the authors to explore the presence of self-created risks for chronic-degenerative diseases, to identify factors related to health promotion and disease prevention as well as to reflect about workers' self-care and self-responsibility concerning their health, aiming at the quality of life they will enjoy as a result of the demographic and epidemiological transition that we are currently experiencing.

DESCRIPTORS: public health nursing, health promotion, workers in health care units

\section{Perfil de salud de trabajadores de Un Centro de salud escuela}

El presente trabajo buscó describir el perfil de salud de un conjunto de trabajadores (184) de un Centro de Salud Escuela de Ribeirão Preto, sob la perspectiva de la Promoción de la salud, modelo epidemiológico de Blum y factores de riesgo para enfermedades crónico-degenerativas Fue realizado un estudio descriptivo usando la entrevista estructurada. Se concluye que los trabajadores eran predominantemente del sexo femenino, rango de edad entre 35 y 39 años, escolaridad "superior completa". Se constató que $61,9 \%$ estaban con sobrepeso y obesidad; la hipertensión fue detectada en 33,1\%; 41 (22,3\%) presentaron presión arterial por encima del nivel normal y la obesidad se mostró presente en mas de la mitad de los individuos con presión arterial alterada. Los datos posibilitaron explorar la presencia de riesgos autocreados para las enfermedades crónico-degenerativas; identificar factores relacionados con la promoción de la salud y prevención de enfermedades y reflexionar sobre el autocuidado y la autoresponsabilidad, buscando la calidad de vida que disfrutaran debido a la transición demográfica y epidemiológica que estamos viviendo.

DESCRIPTORES: enfermería en salud pública, promoción de la salud, trabajador de puestos de salud

\footnotetext{
${ }^{1}$ Parte da dissertação de mestrado apresentada ao Programa de Pós-Graduação da Escola de Enfermagem de Ribeirão Preto, da Universidade de São Paulo, área: Enfermagem de Saúde Pública; ${ }^{2}$ Enfermeira, Professor Assistente, Doutoranda do Programa Interunidades de Doutoramento em Enfermagem/Escola de Enfermagem de Ribeirão Preto, e-mail: lucila@eerp.usp.br; ${ }^{3}$ Enfermeira, Professor Associado e Orientadora. Escola de Enfermagem de Ribeirão Preto, da Universidade de São Paulo, Centro Colaborador da OMS para o desenvolvimento da pesquisa em Enfermagem
} 


\section{INTRODUÇÃO}

As rápidas e profundas modificações econômicas, políticas e sociais ocorridas de forma heterogênea nas diversas regiões do Brasil, a partir da década de 60 , resultaram em mudanças marcantes na sociedade, caracterizadas por fatores como a diminuição acentuada do índice de fertilidade, redução da mortalidade por doenças infecciosas e parasitárias e aumento expressivo da esperança de vida ao nascer, e modificou-se, também, o estilo de vida da população, com apreensão ou reforço de hábitos, muitas vezes indesejáveis à saúde ${ }^{(1)}$.

O declínio das taxas de fecundidade no Brasil, nas últimas décadas, trouxe grandes implicações sobre a tendência demográfica de sua estrutura etária, apontando para um crescimento importante do número de idosos no país e configurando outro perfil populacional, com conseqüente necessidade de novo delineamento de prioridades $^{(2-4)}$

Paralela a essa transição demográfica, vivenciamos a transição epidemiológica que consiste na alteração do perfil de morbimortalidade do país, decorrente da substituição das causas de morte, anteriormente conseqüentes de doenças infecciosas e parasitárias, que atingem mais a população infantil, pelas doenças crônico-degenerativas, freqüentes na população adulta $^{(4-5)}$.

Nesse novo quadro demográfico e epidemiológico, indivíduos atingem um período da vida quando problemas decorrentes de doenças crônicodegenerativas tornam-se manifestos, somando-se às diferentes condições de vida das pessoas idosas no Brasil, já que são diretamente afetadas por desigualdades de renda, educação, habitação, transporte, acesso aos serviços de saúde, dentre outros. Portanto, torna-se evidente nossa preocupação com a qualidade de vida dessas pessoas que alcançarão a velhice.

Dentre as doenças crônico-degenerativas de maior relevância epidemiológica, destacam-se as doenças cardiovasculares, que incluem a doença isquêmica do coração e as doenças cérebro-vasculares, neoplasias, hipertensão arterial, diabetes mellitus, doença pulmonar obstrutiva crônica, cirrose e as lesões por acidentes e violência ${ }^{(6-7)}$.

O controle dos indicadores de prevalência, incidência e mortalidade para as doenças crônico- degenerativas só pode ser alcançado através de esforços concentrados na prevenção dos fatores de risco associados a esses agravos ${ }^{(8-9)}$. Agir, levando-se em conta tais fatores de risco, significa essencialmente falar em prevenção e promoção de saúde, com o planejamento das ações e recursos voltados para as pessoas, enquanto indivíduos e coletividade.

Para a doença arterial coronariana, os fatores de risco mais importantes têm sido atribuídos à hipercolesterolemia, hipertensão arterial e tabagismo ${ }^{(10-}$ 12). Associações também têm sido feitas entre a obesidade, sedentarismo, alterações lipídicas, estresse emocional e hipertensão arterial, com o aumento da morbimortalidade para as doenças crônicodegenerativas, atribuindo-se a cada fator diferentes impactos sobre essas doenças ${ }^{(7,10-11,13-14)}$.

Outros fatores de risco também têm sido apontados como relacionados à hipertensão e às doenças crônico-degenerativas, tais como os aspectos alimentares incorporados pela vida moderna, como a alta ingestão de açúcar refinado e café, baixa ingestão de fibras, peixe e óleos insaturados, uso de anticoncepcionais hormonais e outros fatores psicológicos como a insatisfação no trabalho e no casamento, dificuldades financeiras, ausência de apoio familiar e social e baixo nível ou classe social $^{(14-16)}$.

Considerando a relevância do panorama exposto, ações direcionadas à promoção de saúde e às práticas de prevenção desses fatores de risco são indispensáveis se pretendemos vislumbrar uma boa qualidade de vida ao grupo populacional emergente.

Dentro desta realidade e refletindo sobre a qualidade de vida dos adultos de hoje, nossa futura população idosa, questionamo-nos a respeito de como serão estes idosos de amanhã, pois bem sabemos que viver mais não significa viver melhor. A qualidade de vida vai além de experimentar a longevidade; engloba "o viver que é bom e compensador nas áreas social, afetiva, profissional e a que se refere à saúde"(17).

Avançando nesse raciocínio, instiga-se a necessidade de conhecer como o adulto de hoje está construindo sua saúde; como ele vem "cuidando" de si mesmo, dos seus e do meio ambiente que o rodeia. Nosso enfoque do cuidar extrapola as questões individuais e assume uma importância ainda maior, compreendendo o ser humano e sua influência na saúde individual e socioecológica. $\mathrm{O}$ autocuidado deixa de ser 
reduzido aos aspectos de prevenção de enfermidades e assume parceria com todos os conhecimentos e ciências disponíveis na natureza. Já em relação à saúde, compreendemos que ela é construída e vivida pelas pessoas no cotidiano de cada uma delas. É, também, construída pelo cuidado de cada um consigo mesmo, com os outros e com o meio ambiente, pela habilidade de decidir e de ter controle sobre as circunstâncias da própria vida e pela luta para que a sociedade dê condições que possibilitem a obtenção da saúde por todos os seus membros.

Acreditando que a promoção de saúde constituise em uma ferramenta essencial para o desenvolvimento sustentável e que é um dos caminhos para responder às ameaças emergentes à saúde e, no aspecto mais amplo, à crise global pela qual passamos, optamos por esse referencial.

Nesse sentido, em busca de um modelo que se aproximasse dos princípios contidos na abordagem da promoção de saúde, adotamos o modelo epidemiológico proposto por Blum ${ }^{(18)}$ e, por meio dele, propomo-nos analisar os fatores subjacentes à saúde da população em estudo.

Diante do exposto, os objetivos deste estudo foram descrever o perfil de saúde dos trabalhadores de um Centro de Saúde Escola e identificar características pessoais, associadas à prática de comportamentos relacionados ao autocuidado dos trabalhadores de um Centro de Saúde-Escola.

\section{METODOLOGIA}

Delineamento do estudo

O estudo se caracterizou como sendo do tipo descritivo, de corte transversal.

Local do estudo

A investigação foi realizada em uma das Unidades do Centro de Saúde Escola (CSE) - CSE Sumarezinho - localizada na zona oeste da cidade de Ribeirão Preto-SP, a qual se configura como Unidade Básica e Distrital de Saúde (UBDS).

População estudada

A população da pesquisa constituiu-se do universo de trabalhadores do CSE Sumarezinho, os quais mantinham vínculo empregatício com a instituição, encontravam-se exercendo suas atividades profissionais no período de dezembro de 1997 a fevereiro de 1998 e, concordaram em participar do estudo, totalizando 184 indivíduos.

Procedimento para coleta de dados

Instrumento de coleta de dados

O roteiro utilizado para a coleta de dados da pesquisa foi elaborado com base nos pressupostos da Promoção da Saúde do Adulto ${ }^{(19-20)}$, dos quatro elementos (estilo de vida, meio ambiente, biologia humana e organização dos serviços de saúde) que compreendem o modelo epidemiológico adotado ${ }^{(18)}$ e dos principais fatores de risco para as doenças crônicodegenerativas $^{(7,10-14,21)}$.

Dessa forma, o instrumento constou dos seguintes itens:

I- Identificação do sujeito: nome, sexo e idade.

II- Dados sociais e ambientais: escolaridade, ocupação, satisfação no trabalho, presença de companheiro, número de filhos, participação religiosa e comunitária.

III- Dados referentes ao estilo de vida: padrões alimentares, de eliminação, atividade física, sono e repouso, estresse, tabagismo e consumo de álcool.

IV- Dados relacionados à biologia do indivíduo: dados antropométricos, aferições da pressão arterial, identificação de doenças já instaladas, aspectos referentes à prevenção de doenças e riscos ocupacionais. V- Dados concernentes à organização dos serviços de saúde: compreenderam aspectos relacionados ao tipo de serviço de saúde que o trabalhador procura quando necessita e à utilização de medicamentos.

$\mathrm{O}$ instrumento foi submetido a oito juízes, todos trabalhadores da Escola de Enfermagem de Ribeirão Preto - USP. Na ocasião, foram realizadas as adequações do instrumento, procedendo-se as sugestões dos referidos juízes.

Operacionalização da Coleta de Dados e Considerações Éticas

A princípio, o projeto de pesquisa foi encaminhado à Comissão de Ética Médica do CSE 
Sumarezinho e, após sua aprovação, iniciamos a coleta de dados, ocorrida no período de dezembro de 1997 a fevereiro de 1998.

Após apresentação do entrevistador, os trabalhadores foram informados quanto aos objetivos do estudo, sigilo das informações e foram questionados quanto à disponibilidade de participação. Após o consentimento (consentimento informado), foram realizadas as entrevistas individualmente, nas dependências do CSE Sumarezinho, respeitando-se a disponibilidade do entrevistado.

Os valores da pressão arterial foram identificados através de duas medidas consecutivas, com intervalo mínimo de um minuto entre cada uma, respeitando-se passos e critérios ${ }^{(22-23)}$. A medida foi realizada no braço direito, com o trabalhador sentado e ao final da entrevista, de modo a garantir o repouso necessário para iniciar o procedimento. Utilizou-se esfigmomanômetro do tipo aneróide, previamente testado e calibrado. Dispúnhamos de manguitos com bolsa de borracha de três larguras diferentes: $8,5 \mathrm{~cm}, 12,0 \mathrm{~cm}$ e 15,0 cm. Após a medida da circunferência braquial do trabalhador, selecionava-se a bolsa de borracha com largura adequada ou mais próxima. A circunferência braquial e a largura da bolsa de borracha utilizada foram registradas em impresso próprio. Para a análise dos dados considerou-se o valor de pressão arterial obtido pela média dos valores nas duas medidas.

Os dados antropométricos constituíram-se das medidas do peso e altura dos trabalhadores. Foram realizados sempre após a aferição dos valores da pressão arterial, utilizando-se balança da própria instituição, marca Filizola, previamente calibrada. Para a verificação do peso os trabalhadores permaneceram descalços, sem uso de casacos ou agasalhos. Os valores obtidos foram registradas em quilograma, com aproximação de uma casa decimal.

A estatura foi verificada com os profissionais eretos e com os calcanhares unidos. Utilizou-se para isso uma régua antropométrica. Os valores foram registrados em centímetros, com aproximação de uma casa decimal.

$O$ índice de massa corporal (IMC), eleito para avaliar a obesidade dos trabalhadores em estudo, foi calculado como sendo o peso $(\mathrm{em} \mathrm{Kg})$ dividido pela altura ao quadrado (em metros), eliminando-se, assim, a dependência do tamanho do esqueleto ${ }^{(24)}$.

\section{Processamento e análise dos dados}

Após a coleta dos dados, os mesmos foram transportados para uma base de banco de dados, formatado no Programa FOX PRO vs $2.0^{(25)}$ para microcomputador.

Para o processamento e análise dos dados, utilizou-se o módulo "Analysis", do Programa EPIINFO vs $6.04 \mathrm{c}$ para microcomputadores ${ }^{(26)}$ e os referenciais teóricos da promoção de saúde, do modelo epidemiológico ${ }^{(18)}$ adotado e dos fatores de risco para as doenças crônico-degenerativas.

\section{RESULTADOS}

Quanto à caracterização da população em estudo:

- $62,5 \%$ eram do sexo feminino e $37,5 \%$ do masculino; - a faixa etária de maior freqüência, para ambos os sexos, compreende o início dos anos dos adultos de meia-idade (35 a 39 anos), destacando-se a escolaridade "superior completo" $(41,3 \%)$;

- predomina a categoria funcional de "nível médio de enfermagem" $(32,1 \%)$ e "médicos $(26,6 \%)$, havendo maior concentração de trabalhadores na área assistencial $(71,2 \%)$ que na de apoio;

- $72,8 \%$ dos entrevistados vivia com algum companheiro e tinha dois filhos $(33,2 \%)$;

- a participação em grupo ou comunidade foi revelada pela maioria $(55,4 \%)$, enquanto práticas em direção ao aspecto da espiritualidade. Nesse aspecto, destacamos que uma das estratégias da promoção de saúde, além de decisão política, é a participação da comunidade em ações concretas na definição de estratégias, implementação e tomada de decisão, visando à melhoria das condições de saúde.

Em relação à biologia dos trabalhadores:

- 38,1\% dos indivíduos apresentaram índice de massa corporal na faixa de normalidade, havendo mais mulheres que homens nessa condição; $26,6 \%$ estavam com sobrepeso e $35,3 \%$ com obesidade já instalada, sendo que, nessas duas últimas categorias, prevaleceu o sexo masculino;

- as categorias de Índice de Massa Corporal "sobrepeso" e "obesidade" totalizaram $61,9 \%$ dos entrevistados, com maior prevalência do sexo masculino e idade entre $35 \mathrm{e}$ 40 anos; 
- quanto à pressão arterial, 93 trabalhadores (50,5\%) encontraram-se nos níveis "ideal" e "normal". A associação entre os trabalhadores $(n=41)$ que se apresentaram na faixa de pressão arterial acima do nível de normalidade $(22,3 \%)$ e obesidade foi expressiva, ou seja, tanto para a hipertensão sistólica, quanto para a diastólica, a obesidade, estava presente para mais da metade dos entrevistados;

- a hipertensão foi mais freqüente no sexo masculino e totalizava 61 indivíduos (33,1\%), com idades entre $42 \mathrm{e}$ 45 anos. A obesidade foi detectada tanto entre aqueles com hipertensão sistólica, quanto diastólica, em mais de $60 \%$ dos trabalhadores;

- a hipertensão arterial foi a doença instalada mais citada pelos trabalhadores $(15,8 \%)$;

- o auto-exame das mamas e a realização de exame preventivo do câncer do colo do útero, apesar de terem sido referidos pela maioria $(63,5 \%$ e $87,0 \%$, respectivamente) das mulheres entrevistadas, revelou ausência dessas práticas em uma expressiva parcela dessa população (36,5\% e 13,0\%, respectivamente) constituindo riscos à sua saúde;

- a prevenção do câncer de próstata foi afirmada em apenas $28,6 \%$ dos homens situados na faixa etária recomendada para esse fim, apresentando, também, aspecto negativo à promoção de saúde. Os resultados do estudo sugerem, ainda, o desenvolvimento de práticas educativas com os trabalhadores a fim de prevenir o câncer de pele, pois, apesar da baixa mortalidade, é o câncer mais incidente no Brasil, e o uso de protetor solar foi relatado pela maioria $(51,6 \%)$, apenas quando expostos ao sol;

- a imunização contra o tétano e hepatite B não se encontravam atualizadas para $15,2 \%$ e $35,9 \%$, respectivamente, das populações em estudo;

- Os riscos ocupacionais mostraram-se presentes através do relato de $50,5 \%$ dos trabalhadores que haviam tido acidentes de trabalho, sugerindo alguma relação entre a "ausência" e "satisfação em parte" dos indivíduos em relação ao seu trabalho $(41,0 \%)$.

Quanto ao estilo de vida dos indivíduos:

- hábitos alimentares: $91,3 \%$ dos trabalhadores ingeriam todos os tipos de carne; $45,7 \%$ consumiam ovos até três vezes por semana, e 41,8\% sob a forma de ovo oculto; a fritura era consumida por $37 \%$ dos trabalhadores, mais de quatro vezes por semana, e a gordura mais utilizada no preparo dos alimentos foi a de origem vegetal; diariamente, doces eram consumidos por $32,1 \%$ dos trabalhadores, e $30 \%$ ingeriam refrigerantes com a mesma freqüência; o consumo diário de café foi relatado por $81,5 \%$ dos indivíduos, sendo que $31,5 \%$ ingeriam mais de quatro xícaras diárias; a água era consumida em volume inferior ao recomendado, por mais de $80 \%$ dos trabalhadores; o hábito de consumir fibras, avaliado através da ingestão de frutas e verduras, foi freqüente para a maioria dos entrevistados, embora se possa inferir, com base na literatura, que a quantidade consumida deva ser aquém da recomendada. Apesar dos benefícios, havia trabalhadores que, raramente ou nunca, consumiam frutas e verduras, caracterizando suas dietas como deficientes nesses nutrientes;

- a adição de sal à mesa não constituiu hábito negativo relatado pela maioria $(87,5 \%)$ dos trabalhadores, apesar de $12,5 \%$ apresentar esse costume. Dentre os trabalhadores identificados como hipertensos em nosso estudo, 14,8\% afirmaram esse hábito, apresentando-se como fato limitante ao controle da hipertensão e aumentando o risco para outras doenças cardiovasculares;

- padrões de eliminação: $75,5 \%$ dos indivíduos não afirmaram constipação intestinal, referindo hábito de evacuação diária, e os demais, $24,5 \%$, relataram ser constipados. O uso de laxante fez-se presente por $33,3 \%$ dos trabalhadores constipados, apesar de a maioria referir consumo diário de frutas e verduras, sugerindo quantidade insuficiente de ingestão por dia;

- sistema urinário: nove mulheres relataram incontinência urinária, e um homem, dificuldade para urinar. Idade avançada, vida sedentária e número de filhos revelaram-se enquanto contribuintes no processo de incontinência urinária nas mulheres;

- a atividade física não era desenvolvida por mais da metade dos entrevistados, sugerindo participação nos altos índices de sobrepeso e obesidade encontrados na população estudada;

- o hábito do fumo foi referido por $22,3 \%$ dos trabalhadores, e o do álcool, por 46,2\%, constituindo oportunidade para atuarmos objetivando a prevenção primária e agindo para minimizar os efeitos da concomitância de fatores de riscos comumente presentes no estilo de vida destes indivíduos;

- padrões de sono e descanso; estresse e uso de tranqüilizantes: a necessidade de descanso não foi revelada pela maioria dos entrevistados (70\%). O 
estresse foi referido por quase metade da população estudada (47,3\%), sendo que o ambiente do trabalho foi o considerado mais estressante pelos trabalhadores $(46,7 \%)$, revelando-se o ambiente de trabalho como um dos fatores importantes na determinação de saúde dos entrevistados. 0 uso de tranqüilizantes não era feito por $93,5 \%$ dos entrevistados. Dentre aqueles que os utilizavam (6,5\%), metade praticava a automedicação.

Dados relacionados ao atendimento de saúde:

- quanto à utilização dos serviços de saúde, o do tipo privado foi o mais citado (43\%), seguido de perto pela sua associação com o público (38\%), e o uso de medicamentos não se constituiu como prática realizada por mais da metade dos trabalhadores, porém a automedicação, mesmo que pouco expressiva, mostrou-se presente para quase $10 \%$ dos trabalhadores.

\section{CONSIDERAÇÕES FINAIS}

Os resultados evidenciaram a presença de riscos autocriados, doenças já instaladas e aspectos da biologia dos trabalhadores que também se mostraram potencialmente capazes de agir de forma negativa sobre os determinantes de sua saúde.

É interessante comentar que o fato de se tratar de uma instituição onde mais da metade dos trabalhadores lidam diretamente com o público, educando e cuidando dos aspectos de sua saúde, não exime os trabalhadores dos fatores de risco para as doenças não transmissíveis, que acompanham a transição epidemiológica de nossos tempos.

Apesar de identificarmos aspectos intensificadores de saúde na população estudada, sua freqüência não é suficiente para nos tranqüilizarmos quanto à qualidade de vida que esses futuros adultos idosos desfrutarão, em decorrência da mudança do padrão demográfico que já nos rodeia. A autoresponsabilidade e o autocuidado constituem-se habilidades essenciais para a superação desses entraves. E é nessa direção que a promoção de saúde e a educação fazem-se instrumentos dos indivíduos que buscam o controle da própria saúde, assim como de seus determinantes. Na complementaridade, a visão sistêmica oferece suporte à promoção de saúde, em busca de um desenvolvimento sustentável. Imprescindível se faz a necessidade de reconhecimento do indivíduo enquanto sujeito de sua própria vida; responsável pela construção e cuidado de sua saúde, do outro e do meio ambiente que o cerca.

A realização deste estudo teve, como ponto de partida, buscar compreender a realidade dos trabalhadores e, dentro dessa compreensão, considerar que eles se inserem num contexto de país em desenvolvimento, que apresenta desigualdades sociais e dependência econômica. Nesse sentido, a escolha do nosso referencial teórico permitiu-nos contextualizar esses trabalhadores e, para nós, o primeiro passo para qualquer mudança é o conhecimento da própria realidade.

Esse conhecimento, à medida que está disponível e vai sendo incorporado pelos trabalhadores, permite que o saber por meio da consciência ingênua possa ser transcendido pelo saber e fazer conscientizado, refletindo, tomando decisões e assumindo riscos. Entendemos que é necessário começar pelo menos pela consciência da necessidade, para, então, de posse do saber dos determinantes de nosso mundo, avaliarmos as possibilidades de transformação da situação de que dispomos. Cabe aqui lembrar a importante capacidade criativa do ser humano.

Só podemos enxergar o outro por meio de nós mesmos e, por isso, precisamos, a princípio, enxergarnos, saber nosso lugar no mundo e as possibilidades que se nos apresentam. Conhecendo-se a si próprio, o homem pode tornar-se agente transformador de sua realidade e da realidade maior que o engloba. É o grande salto da transformação da heteronomia que nos envolve, para a autonomia, que pode ser alcançada através da Promoção de Saúde, já que apresenta, como uma de suas ferramentas, a prática do autocuidado, entendida como uma das formas de expressão dessa autonomia do indivíduo.

A possibilidade de realizarmos tentativas de desconstrução de práticas inconscientes em relação à saúde e partirmos para construção de hábitos saudáveis, com auto-responsabilidade, autocuidado e autonomia, certamente trará benefícios, não só aos trabalhadores, como também à sua clientela, à medida que poderá servir como facilitador de troca de experiências entre ambos, promovendo a saúde e prevenindo doenças. 


\section{REFERÊNCIAS BIBLIOGRÁFICAS}

1. Lessa I. Doenças não transmissíveis. In: Rouquayrol MZ. Epidemiologia e saúde. $4^{a}$ ed. Rio de Janeiro (RJ): MEDSI; 1994. p. 269-79.

2. Kalache A, Veras RP, Ramos LR. O envelhecimento da população mundial: um desafio novo. Rev Saúde Pública 1987; 21(3):200-10.

3. Ramos LR, Rosa TEC, Oliveira ZM, Medina MCG, Santos FRG. Perfil do idoso em área metropolitana na região sudeste do Brasil: resultados de inquérito domiciliar. Rev Saúde Pública 1993 abril; 27(2):87-94.

4. Veras RP. País jovem com cabelos brancos: a saúde do idoso no Brasil. $2^{a}$ ed. Rio de Janeiro (RJ): Relume Dumará; 1994.

5. Patarra NL. Mudanças na dinâmica demográfica. In: Monteiro CA. Velhos e novos males da saúde no Brasil: a evolução do país e de suas doenças. São Paulo (SP): HUCITEC/NUPENS - USP; 1995. p. 61-78.

6. Rego RA, Bernardo FAN, Rodrigues SSR, Oliveira ZMA, Oliveira $M B$, Vasconcellos $C$, et al. Fatores de risco para doenças crônicas não-transmissíveis: inquérito domiciliar no município de São Paulo, SP (BRASIL). Metodologia e resultados preliminares. Rev Saúde Pública 1990 agosto; 24(4):277-85.

7. Duncan BB, Schmidt MI, Polanczyk CA, Homrich CS, Rosa RS, Achutti AC. Fatores de risco para doenças não transmissíveis em área metropolitana na região sul do Brasil: prevalência e simultaneidade. Rev Saúde Pública 1993 fevereiro; 27(1):143-8.

8. Feliciano AB. Demanda por doenças crônico-degenerativas entre adultos matriculados em uma unidade básica de saúde em São Carlos-SP. [dissertação]. Ribeirão Preto (SP): Escola de Enfermagem de Ribeirão Preto/USP; 1997.

9. Ortiz MCA, Zanetti ML. Levantamento dos fatores de risco para diabetes mellitus tipo 2 em uma instituição de ensino superior. Rev Latino-am Enfermagem 2001 maio; 9(3):58-63. 10. Guimarães AS. Cardiopatia coronária: aspectos epidemiológicos e preventivos. Arq Bras Cardiol 1992 julho; 59(1):5-11.

11. Stovsky B. Nursing interventions for risk factor reduction. Nurs Clin North Am 1992 March; 27(1):257-70.

12. Chor D, Fonseca MJM da, Andrade CR. Doenças cardiovasculares: comentários sobre a mortalidade precoce no Brasil. Arq Bras Cardiol 1995 janeiro; 64(1):15-9.

13. Cruz DALM da. Aspectos de enfermagem na prevenção primária das doenças isquêmicas do coração. Rev Esc Enfermagem USP 1988 agosto; 22(2):215-21.

14. Mancilha-Carvalho JJ. Antecedentes da doença coronária: os fatores de risco. Arq Bras Cardiol 1992; 58(4):263-7.

15. Ornish D. Salvando o seu coração: o único programa científico para prevenir e reverter doenças do coração, naturalmente, sem cirurgias e sem remédios. Rio de Janeiro (RJ): Relume-Dumará; 1993.

16. Silva MAD. Quem ama não adoece: o papel das emoções na prevenção e cura das doenças. $10^{a}$ ed. São Paulo (SP): Best Seller; 1994.

17. Lipp M, Rocha JC. Você está hipertenso. In: Lipp M, Rocha JC. Stress, hipertensão arterial e qualidade de vida: um guia de tratamento para o hipertenso. Campinas (SP): Papirus; 1994. p. 9-17.
18. Blum HL. Planning for health: generics for the eighties. $2^{a}$ ed. New York: Human Sciences Press; 1981. p. 2-38.

19. Behler D, Tippett T, Mandle CL. Midle adult. In: Edelman $\mathrm{CL}$, Mandle CL. Health promotion: throughout the lifespan. $3^{\mathrm{a}}$ ed. Philadelphia: Mosby; 1994. p. 607-31.

20. Kudzuma EC, Quinn J. Young adult. In: Edelman CL, Mandle CL. Health promotion: throughout the lifespan. $3^{a}$ ed. Philadelphia: Mosby; 1994. p. 573-606.

21. Cunningham $\mathrm{S}$. The epidemiologic basis of coronary disease prevention. Nurs Clin North Am 1992 March; 27(1):15369.

22. Joint National Committee sobre prevenção, detecção, avaliação e tratamento da hipertensão arterial $\left(6^{\circ}\right.$ rel. internacional). Bethesda: National Institutes of Health; 1997. 22. Ministério da Saúde (BR). Secretaria de Assistência à Saúde. Departamento de Programas de Saúde. Coordenação de Doenças Cardiovasculares. Controle da Hipertensão Arterial: uma proposta de integração ensino-serviço. Rio de Janeiro (RJ): CDCV/NUTES; 1993.

24. International Lipid Information Bureau (ILIB) Latino America. Recomendaciones de ILIB para el diagnóstico de las dislipidemias en Latinoamérica. Cardiovasc Risk Factors 1994; 3(1 Suppl 1):10-27.

25. Fox Holdings Inc. Fox pro v. 2.0 Pat Pend American Edition; 1991.

26. Dean J. Epiinfo: computer programs for epidemiology. Atlanta: Division of Surveillance and Epidemiology Studies, Epidemiology Programs Office, Center for Disease Control; 1997. 